\title{
Far Lateral Approach for Malignant, Severely Calcified Cerebellopontine Angle Tumor
}

\author{
Satoshi Kiyofuji $^{11}$ Christopher S. Graffeo ${ }^{1}$ Avital Perry $^{1} \quad$ Michael J. Link ${ }^{1}$ \\ ${ }^{1}$ Department of Neurologic Surgery, Mayo Clinic, Rochester, \\ Minnesota, United States

\begin{abstract}
Address for correspondence Satoshi Kiyofuji, MD, Department of Neurologic Surgery-Mayo Clinic, 200 First Street Southwest, Rochester, MN 55905, United States (e-mail: skiyofu1@gmail.com).
\end{abstract}

J Neurol Surg B 2021;82(suppl S1):S29-S30.

\begin{abstract}
Keywords

- calcified tumor

- cerebellopontine angle tumor

- far lateral approach

- glioblastoma

Background The far-lateral approach is an effective skull base technique that provides access to the lower clivus and premedullary area. This approach is also useful for maximal visualization and resection of large posterior fossa tumors with extensive medial extension, especially when aggressive resection is ideal for a malignant tumor in a young patient, or it is technically challenging because of tumor calcification. We demonstrate a microsurgical operative video to describe technical pearls in this difficult situation.

Case Presentation A 45-year-old man with history of "hoarseness" for up to two decades was presented with imbalance and mild dysphagia over 3 years. Imaging demonstrated a 55-mm left cerebellopontine angle (CPA) tumor extending medially across the midline with severe calcification (-Figs. $\mathbf{1}$ and $\mathbf{2}$ ). His neurological examination revealed left facial numbness, complete left facial weakness, left deafness, complete left vocal paralysis, as well as severe left hemibody ataxia. The tumor was resected via a left suboccipital craniotomy with far lateral approach including drilling to the occipital condyle and C1 laminectomy. Initial manipulation of the inferior pole of the tumor resulted in asystole which was managed successfully with glycol pyrolate. Pathology demonstrated IDH-1 wild type, MGMT-methylated glioblastoma. The patient subsequently underwent adjuvant chemoradiation.

Conclusion The far-lateral approach is an effective approach for maximal safe resection of a malignant brainstem, cerebellar, and CPA tumor.

The link to the video can be found at: https://youtu.be/AIGebJPJEnw.
\end{abstract}

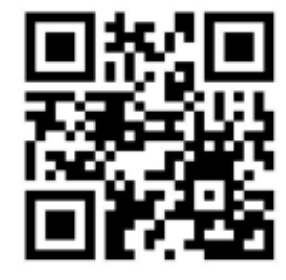

received

February 26, 2019

accepted

DOI https://doi.org/

10.1055/s-0040-1705164. ISSN 2193-6331.
Conflict of Interest

None declared.

www.thieme.com/skullbasevideos

www.thieme.com/jnlsbvideos
January 13, 2020

published online

November 17, 2020
This is an open access article published by Thieme under the terms of the Creative Commons Attribution-NonDerivative-NonCommercial-License, permitting copying and reproduction so long as the original work is given appropriate credit. Contents may not be used for commercial purposes, or adapted, remixed, transformed or built upon. (https://creativecommons.org/ licenses/by-nc-nd/4.0/)

Georg Thieme Verlag KG, Rüdigerstraße 14, 70469 Stuttgart, Germany 
S30 Far Lateral Approach for CPA Tumor Kiyofuji et al.

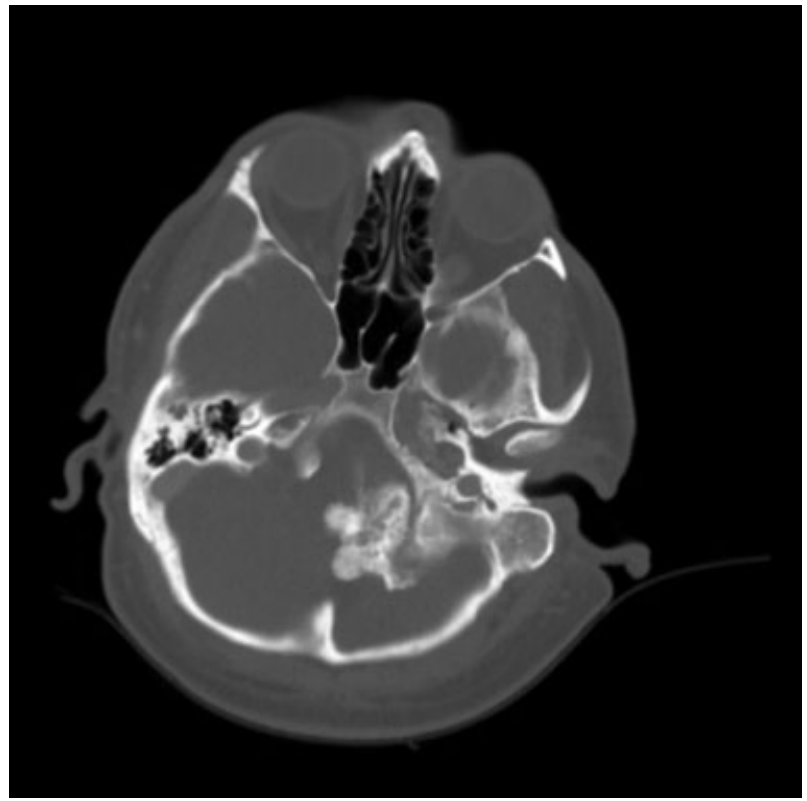

Fig. 1 Preoperative axial image of computed tomography (CT) demonstrates prominently calcified lesion in the left cerebellopontine angle (CPA).

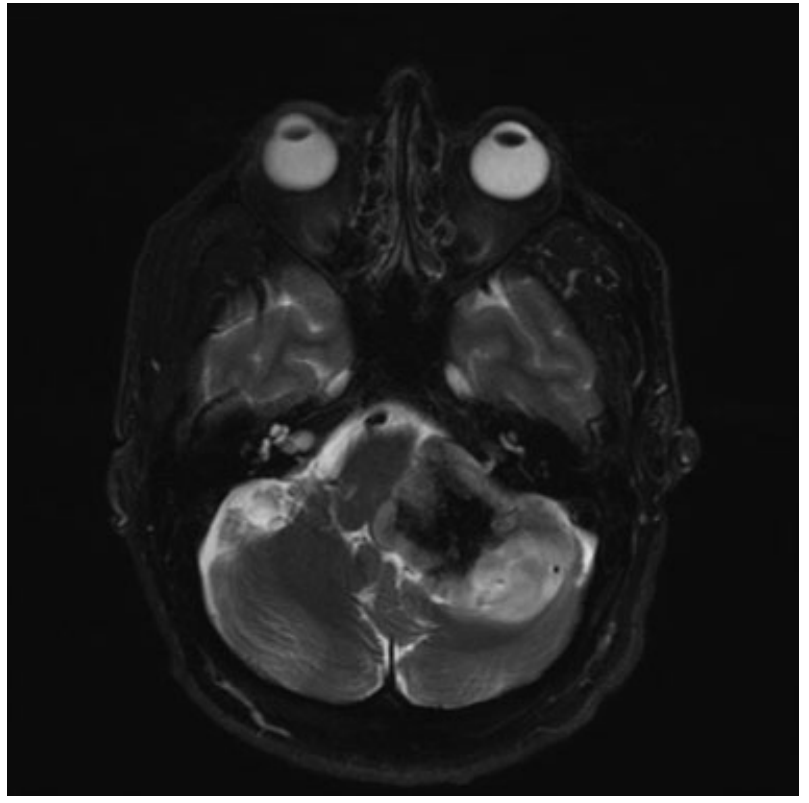

Fig. 2 Preoperative axial T2-weighted image of magnetic resonance imaging (MRI) demonstrates a 55-mm left cerebellopontine angle (CPA) lesion that extends across the midline to displace the medulla contralaterally. 